The BMJ

Cite this as: BMJ 2021;374:n2251 http://dx.doi.org/10.1136/bmj.n2251 Published: 14 September 2021

\title{
Covid-19: Study that claimed boys are at increased risk of myocarditis after vaccination is deeply flawed, say critics
}

Clara Munro

The risk of 12-15 year old healthy boys experiencing cardiac adverse events such as myocarditis after their second dose of the Pfizer and BioNTech vaccine is around four times adolescents' risk of being admitted to hospital as a result of infection with SARS-CoV-2, a preprint study has found. ${ }^{1}$

However, the study has been widely criticised for mining data from an inappropriate source to deliver an antivaccine message, despite warnings against such data uses.

The retrospective study, which has not yet been peer reviewed, used the US vaccine adverse reporting system (VAERS) to identify the rate of

post-vaccination myocarditis among 12-15 and 16-17 year olds between January and June 2021 after the second dose of the Pfizer-BioNTech vaccine. The researchers concluded that the rate of cardiac adverse events after the second dose exceeded the expected rate of 120 day covid-19 hospital admission at both a moderate (August 2021) and a high (January 2021) incidence of SARS-CoV-2 infection.

They estimated that the rate of cardiac myocarditis after the second dose of the vaccine was 162.2 cases per million in healthy boys aged 12-15 years and 94.0 per million in those aged 16-17. For girls the rate was 13 per million in 12-15 year olds and 13.4 per million in $16-17$ year olds. Nearly all (86\%) of the boys affected needed some form of hospital care, the authors said. By comparison, at current infection rates the risk of a healthy adolescent being admitted to hospital within 120 days of infection in the US is estimated to be 44 per million.

Trish Greenhalgh, professor of primary care health sciences at the University of Oxford, told The BMJ that although all preprints were suspect before they were peer reviewed, some were more suspect than others.

She said, "The VAERS database is a passive monitoring system maintained by the US Food and Drug Administration and Centers for Disease Control and Prevention that invites the public to report any perceived or suspected side effects following vaccination, so that potential signals of harm may be investigated further. Crucially, all such reports must be validated by other active monitoring systems, as VAERS entries are very prone to reporting and recall bias.

"Indeed, the CDC explicitly states that VAERS cannot be used in isolation to infer the existence, frequency, or rates of vaccine complications."

Greenhalgh said that although the FDA and CDC used VAERS data to generate hypothesis driven questions about effects of covid vaccines in teenagers, the agencies then investigated before concluding that these vaccines were safe.

"VAERS data dredging, as it is known, has been used by antivaccine groups in the past to produce alarmist estimates of harms from vaccines," she said.

In a blog post on the website Science-Based Medicine, Dan Freedman, a paediatric neurologist in Austin, Texas, highlighted this and other methodological flaws of the study, including that many of the "cases" of myocarditis may have been from an infection or another diagnosis altogether. ${ }^{2}$ He described the analysis as "half-baked" with "data that will certainly be co-opted by the antivaccine movement."

The US and Israel have been among the first countries to widely vaccinate younger people and so preliminary data on vaccine complications in these groups are becoming more readily available.

A large Israeli study published in the New England Journal of Medicine showed an excess risk of myocarditis in people over 16 receiving the Pfizer-BioNTech vaccine (1-5 events per 100000 persons). ${ }^{3}$ While demographic information was not specifically analysed, it was notable that 19 of the 21 myocarditis cases were in males, with the median age of those affected being 25 .

In June the US Centers for Disease Control and Prevention's safety committee said there was a "likely association" between the Pfizer-BioNTech and Moderna covid vaccines and myocarditis and pericarditis in some young adults, but the US Department of Health and Human Services and other agencies emphasised that the side effect was "extremely rare" and that most cases were mild. ${ }^{4}$

The UK's Medicines and Healthcare Products Regulatory Agency said it had come to the same conclusions: cases of myocarditis and pericarditis were rare and tended to be mild, with most affected people recovering with simple treatment and rest.

The study's authors defended their use of the VAERS database and their selection of cases. Coauthor Allison Krug told The BMJ, "Because VAERS is a national surveillance system with a simple reporting mechanism, it yields timely information and has high sensitivity for new vaccine safety concerns."

Coauthor Tracy Beth Høeg added, “We went through all entries to ensure there was objective evidence of cardiac damage and that these were not self-reports of just 'chest pain' but required the same objective evidence of cardiac injury that the CDC did in their analysis."

Krug said that as they revised their study the team would be looking at comparing rates of hospital 
admission for myocarditis with admission rates for covid infection.

Correction: On 17 September this news story was updated in the light of criticism of the preprint being reported. On 21 September we added the final three paragraphs giving the coauthors' responses.

1 Høeg TB, Krug A, Stevenson J, Mandrola J. SARS-CoV-2 mRNA vaccination-associated myocarditis in children ages 12-17: a stratified national database analysis.MedRxiv 2021.08.30.21262866 [Preprint]. https://www.medrxiv.org/content/10.1101/2021.08.30.21262866v1.

2 Freedman D. Peer review of a VAERS dumpster dive. Science-Based Medicine. Sep 2021. https://sciencebasedmedicine.org/peer-review-of-a-vaers-dumpster-dive.

3 Barda N, Dagan N, Ben-Shlomo Y, etal. Safety of the BNT162b2 mRNA Covid-19 Vaccine in a Nationwide Setting. N Engl J Med 2021. doi: 10.1056/NEJMoa2110475. pmid: 34432976

4 Wise J. Covid-19: Should we be worried about reports of myocarditis and pericarditis after mRNA vaccines?BM/2021;373:n1635. doi: 10.1136/bmj.n1635 pmid: 34167952

This article is made freely available for use in accordance with BMJ's website terms and conditions for the duration of the covid-19 pandemic or until otherwise determined by BMJ. You may use, download and print the article for any lawful, non-commercial purpose (including text and data mining) provided that all copyright notices and trade marks are retained. 